\title{
Studying the interaction between microquasar jets and their environments
}

\author{
M. Perucho \\ Max-Planck-Institut für Radioastronomie, \\ Auf dem Hügel, 69, Bonn 53121, Germany \\ E-mail: perucho@mpifr-bonn.mpg.de \\ V. Bosch-Ramon \\ Max Planck Institut für Kernphysik \\ Saupfercheckweg 1, Heidelberg 69117, Germany \\ E-mail:vbosch@mpi-hd.mpg.de
}

\begin{abstract}
In high-mass microquasars (HMMQ), strong interactions between jets and stellar winds at binary system scales could occur. In order to explore this possibility, we have performed numerical 2-dimensional simulations of jets crossing the dense stellar material to study how the jet will be affected by these interactions. We find that the jet head generates strong shocks in the wind. These shocks reduce the jet advance speed, and compress and heat up jet and wind material. In addition, strong recollimation shocks can occur where pressure balance between the jet side and the surrounding medium is reached. All this, altogether with jet bending, could lead to the destruction of jets with power $<10^{36} \mathrm{erg} / \mathrm{s}$. The conditions around the outflow shocks would be convenient for accelerating particles up to $\sim \mathrm{TeV}$ energies. These accelerated particles could emit via synchrotron and inverse Compton (IC) scattering if they were leptons, and via hadronic processes in case they were hadrons.
\end{abstract}

Keywords: X-rays: binaries - stars: individual: LS 5039 - Radiation mechanisms: non-thermal

\section{Introduction}

The interaction between a jet and its environment allows the study of both the properties of the jet and its ambient medium. ${ }^{1,2}$ Moreover, this interaction is important for the evolution of jets. ${ }^{3}$ In the case of extragalactic jets, it is known to be different in case of FRI and FRII sources; the collision and interaction of jets with galactic clouds and collective stellar winds is thought to be partly responsible for the jet mass loading and deceleration in the former, ${ }^{4}$ whereas the latter can propagate and interact with the medium up to Mpc scales. In the case of microquasars, not much is known about the interaction of jets with their environments, ${ }^{5,6}$ and only several sources show evidence of such an interaction (e.g. SS $433 ;{ }^{7}$ XTE J1550-564; ${ }^{8}$ Cygnus X-1 ${ }^{9}$ ).

In HMMQ, the primary star suffers severe mass loss in the form of a supersonic wind, ${ }^{10}$ which can embed the jet rendering their mutual interaction likely. Here we report on a work studying this interaction and its effects on the jet evolution and radiation.

\section{Numerical Simulations}

We have performed three numerical simulations of jets with cylindrical geometry and two with slab (i.e. planar $^{\mathrm{a}}$ ) geometry propagating through the wind region. We simulate the injection of a jet, with the properties given in Table 1, in the ambient medium, i.e. the stellar wind. The wind parameters correspond to those of a HMMQ similar to Cygnus X-1 or LS 5039. The density is $2.8 \times 10^{-15} \mathrm{~g} / \mathrm{cm}^{-3}$, for a star with $d M_{\mathrm{w}} / d t=10^{-6} \mathrm{M}_{\odot} / \mathrm{yr}$ at $3 \times 10^{12} \mathrm{~cm}$ from the jet base. For simplicity, at this stage the wind porosity (e.g. Ref. 11) is assumed to be negligeable. Typically, the velocity of an $\mathrm{O}$ star wind is $\sim 2 \times 10^{8} \mathrm{~cm} / \mathrm{s}$. Despite this, the ambient is considered to be at rest in the cylindrical simulations, this fact being, in principle, justified because the velocity of the jet is about 100 times larger that the wind one. It allows an axisymmetric treatment of the problem though the real wind is moving and comes from one side, being thus intrinsically asymmetric. We note that this assumption is not valid for weak jets on the basis of the results of the slab geometry simulations. The internal energy of this medium is obtained from

\footnotetext{
aThe slab jet has the same properties as in the cylindrical case per distance unit in the direction perpendicular to the simulated plane, and it is infinite in such a direction.
} 
the temperature assumed for the wind $\left(10^{4} \mathrm{~K}\right)$, with a resulting pressure of $1.5 \times 10^{-3} \mathrm{erg} / \mathrm{cm}^{-3}$. The adiabatic index of both the ambient and the jet (supersonic and weakly relativistic), is $5 / 3$. The parameters in the jets are summarized in Table 1.

Table 1. Parameters of the simulations

\begin{tabular}{llll}
\hline Parameter & Weak j. & Mild j. & Powerful j. \\
\hline Jet power $\left(\mathrm{erg} \mathrm{s}^{-1}\right)$ & $3.0 \times 10^{34}$ & $10^{36}$ & $3.0 \times 10^{37}$ \\
Jet pressure $\left(\mathrm{erg} \mathrm{cm}^{-3}\right)$ & 9.1 & 68 & $6.2 \times 10^{4}$ \\
Jet density $\left(\mathrm{g} \mathrm{cm}^{-3}\right)$ & $2.2 \times 10^{-16}$ & $5.9 \times 10^{-16}$ & $1.8 \times 10^{-14}$ \\
Jet speed $\left(\mathrm{cm} \mathrm{s}^{-1}\right)$ & $1.3 \times 10^{10}$ & $2.2 \times 10^{10}$ & $2.2 \times 10^{10}$ \\
\hline
\end{tabular}

The numerical simulations were performed using a two-dimensional finite-difference code based in a high resolution shock-capturing scheme which solves the equations of relativistic hydrodynamics. This code is an upgrade of that described in Ref. 1. The numerical grid is formed by 320 cells in the radial direction and 2400 cells in the axial direction, with physical dimensions 20x300 jet radii. Extended grids were added in both directions in order to bring the grid boundaries far from the region of interest. The jet is injected at a distance of $6 \times 10^{10} \mathrm{~cm}$ from the compact object, and it is given an initial radius of one tenth of this distance. During the simulation, the jet covers a significant fraction of the typical size of a HMMQ binary system (here we adopt $\left.R_{\text {orb }} \sim 3 \times 10^{12} \mathrm{~cm}\right)$. The planar simulations were performed with the same numerical resolution (which implies doubling the grid radially) and a shorter axial grid by a factor $2 / 3$.

\section{Results}

Figs. 1 and 2 show the mild and powerful jets when reaching the end of the grid, respectively. A large pressure jump $\left(>10^{7}\right)$ is generated at the bow shock in both cases. A reverse shock in the jet itself is also formed. The mild jet generates a high pressure cocoon, which keeps it collimated, whereas the powerful jet develops a strong standing (recollimation) shock, with a pressure jump of the order of $\sim 10^{5}$, due to overpressure with respect to its cocoon. Nevertheless, the mild jet also shows weaker internal shocks with pressure jumps of the order of $\sim 100$. The simulation of a weak jet (not shown here) gives results which are very similar to those of the mild jet.
Dimensional estimates ${ }^{12}$ show that, in the jet/cocoon phase, standing shocks must appear in the binary region $\left(\sim 10^{12} \mathrm{~cm}\right.$ from the jet base), at jet-temperatures $>10^{10} \mathrm{~K}$ at injection in the numerical grid. The temperature of the mild jet is around this limit, which explains the absence of strong shocks. However, the fact that the jet pressure increases with decreasing distance to the compact object would generate also a strong shock in this simulation if the injection point of the jet in the grid were closer to the origin.

In the case of a jet interacting with a stellar wind, the lower limit of the initial jet temperature at which a standing shock would appear is reduced by an order of magnitude. ${ }^{12}$ This is important, as once the jet head is far away, the cocoon will fade out and the stellar wind will take its place in the interaction with the jet, though asymmetrically (only from the direction to the primary star), what would produce asymmetric standing shocks.

We have also performed planar jet simulations for the weak and mild jet, in which the wind velocity is taken into account. Fig. 3 shows the influence of a lateral wind on the weak jet. In these maps, the asymmetrical standing shocks produced by the jet-wind interaction are clearly observed. A second simulation for a slab jet with the same physical parameters as the mild cylindrical jet shows that a jet with power $10^{36} \mathrm{erg} / \mathrm{s}$ can also be destroyed by such a wind. ${ }^{12} 3$-dimensional simulations will be performed in order to check this interaction in a more realistic setup.

\section{Non-thermal radiation from jet-wind interactions}

It is worthy noting the importance of the strong shocks predicted by our simulations from the radiative point of view. On one hand, particle acceleration is likely to take place in the standing shocks or the head of the jet when crossing the denser wind regions. The presence of a non thermal population of electrons plus the strong photon field produced by the primary star will lead to strong IC emission (e.g. Ref. 12,13). Wind-jet interactions may be the mechanism triggering particle acceleration in several massive X-ray binaries with jets from which gamma-rays have been detected. ${ }^{14-16}$ In addition, these shocks will possibly increase also the value of 
the magnetic field (not considered in the simulation at this stage) in the postshocked regions to high values, allowing for efficient synchrotron radiation up to X-rays and beyond (e.g. Ref. 12). Moreover, when enough relativistic proton power were available, there may be significant gamma-ray production via decay of neutral pions from proton-proton interactions due to shock enhancement of the matter density, plus other products like secondary pairs and even neutrinos (e.g. Ref. 17,18). We note that for a $10 \%$ efficiency for energy transferring from shocks to non thermal particles, multiwavelength leptonic luminosities of $\sim 10^{36} \mathrm{erg} / \mathrm{s}$, detectable from low (e.g. radio) to very-high energies (e.g. TeV), may be easily reached in the case of a powerful jet.
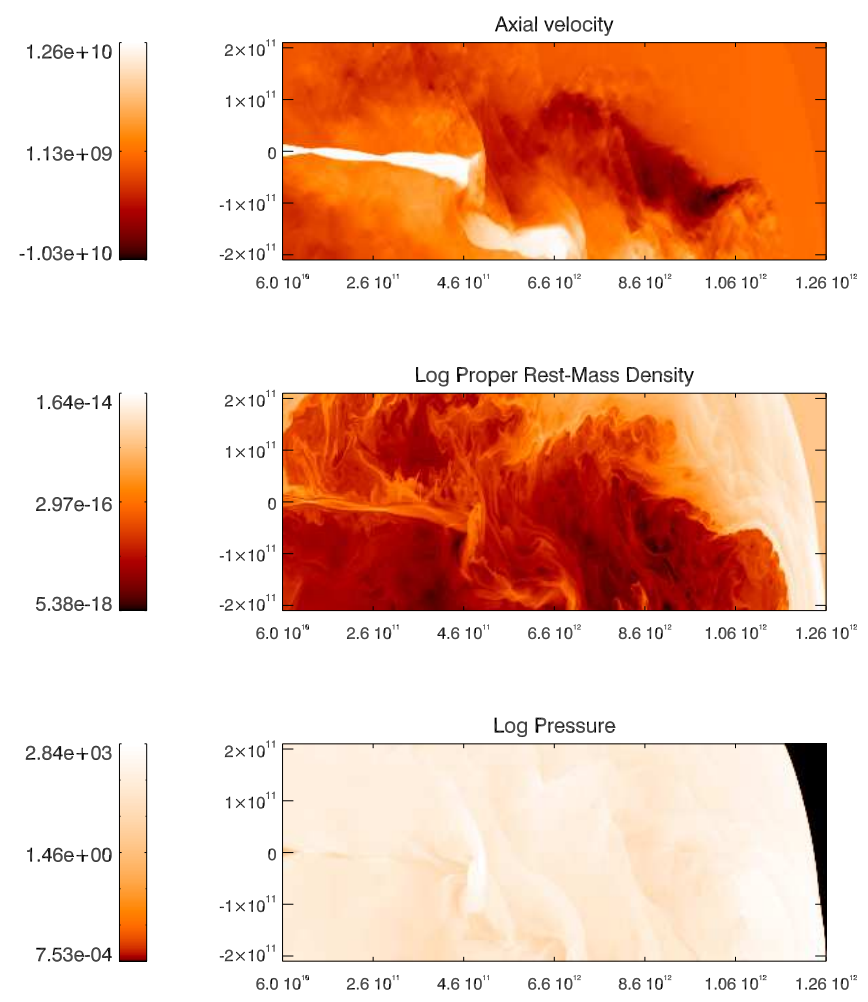

Fig. 3. Maps of axial velocity $\left(\mathrm{cm} \mathrm{s}^{-1}\right)$, rest mass density $\left(\mathrm{g} \mathrm{cm}^{-3}\right)$ and pressure (dyn) and axial velocity $(\mathrm{cm} / \mathrm{s})$ at the end of the simulation $\left(t_{\mathrm{f}}=680 \mathrm{~s}\right)$ of a weak jet in slab geometry. The horizontal and vertical coordinates indicate distances $(\mathrm{cm})$ to the compact object and the jet axis, respectively. The star would be on the vertical axis of the plot, left of the jet, at the orbital distance (out of the plot).

\section{Summary}

We show that, with the reasonable overpressure and physical parameters in the jets and stellar winds of HMMQ, assuming negligible wind porosities, the jets form strong standing shocks due to interaction with the cocoons formed first, and the stellar wind from the primary later, on top of the transient reverse/bow shocks. We estimate that standing shocks must form within the system region for typical jet temperatures $\left(>10^{9-10} \mathrm{~K}\right)$. Moreover, we have shown that jets with power $<10^{36} \mathrm{erg} / \mathrm{s}$ could be deflected and disrupted by the primary star wind. The influence of hydrodynamical instabilities such as Kelvin-Helmholtz is to be neglected on the basis of our results, as the evolution and dynamics of these jets are nonlinear from the start. In this scenario, the inertia of the jet compared to that of the wind is responsible for the fate of the former. Finally, the interaction of the jet head with the wind, and the jet sides with the cocoon and the stellar wind, in HMMQ, could lead to efficient non-thermal multiwavelength radiation, showing the importance of the role of the primary star for high energy emission in these sources, which is not just a provider of seed photons for IC scattering and photon photon absorption, or target nuclei for hadronic processes.

\section{Acknowledgments}

\section{References}

1. Martí, J. M, Müller, E., Font, J.A., Ibáñez, J.M, and Marquina, A. ApJ, 479, 151 (1997).

2. Saxton, C. J., Bicknell, G. V., Sutherland, R. S., Midgley, S. C. J. MNRAS 359, 781 (2005).

3. Perucho, M., Martí, J. M., Hanasz, M. A\&A 443, 863 (2005).

4. Laing, R.A., in Energy Transport in Radio Galaxies and Quasars, eds.: P.E. Hardee, A.H. Bridle and J.A. Zensus, ASP Conference Series, vol. 100, p.241 (1996).

5. Heinz, S. \& Sunyaev, R. A\&A 390, 751 (2002).

6. Bordas, P., Paredes. J. M., Bosch-Ramon, V. these proceedings (2008).

7. Zealey, W. J., Dopita, M. A., \& Malin, D. F. 1974, MNRAS, 192, 731

8. Corbel, S., Fender, R. P., \& Tzioumis, A. K., et al. Science, 298, 196 (2002)

9. Gallo, E., Fender, R., Kaiser, C., et al. 2005, Nature, 436,819

10. Lamers, H. J. G. L. M. \& Leitherer, C. ApJ 412, 771 (1993).

11. Owocki, S. \& Cohen, D. ApJ 648, 565 (2006).

12. Perucho, M. \& Bosch-Ramon, V. $A \mathscr{G} A$ submitted 

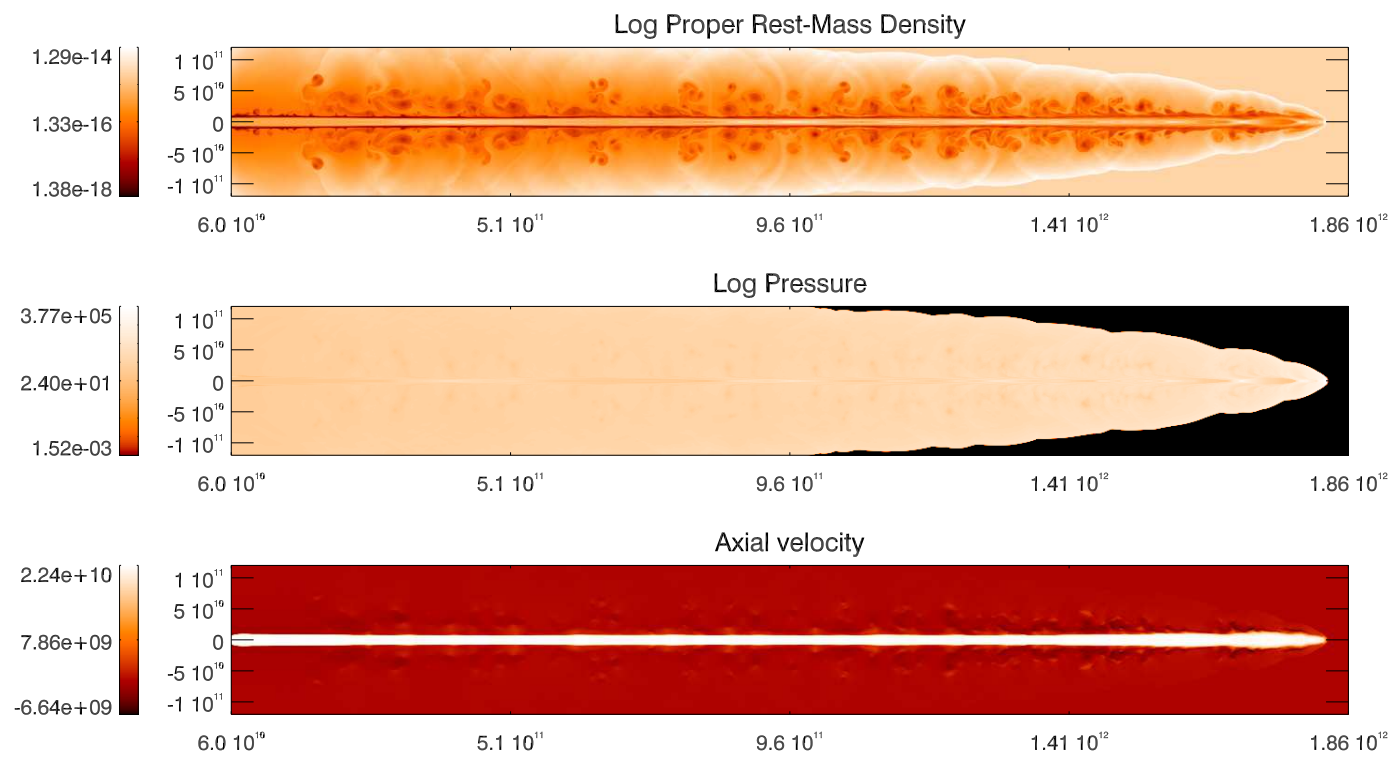

Fig. 1. Maps of rest mass density $\left(\mathrm{g} \mathrm{cm}^{-3}\right)$, pressure $(\mathrm{dyn})$ and axial velocity $(\mathrm{cm} / \mathrm{s})$ at the end of the simulation $\left(t_{\mathrm{f}}=164 \mathrm{~s}\right)$ of the mild jet in cylindric geometry. The horizontal and vertical coordinates indicate distances $(\mathrm{cm})$ to the compact object and the jet axis, respectively.

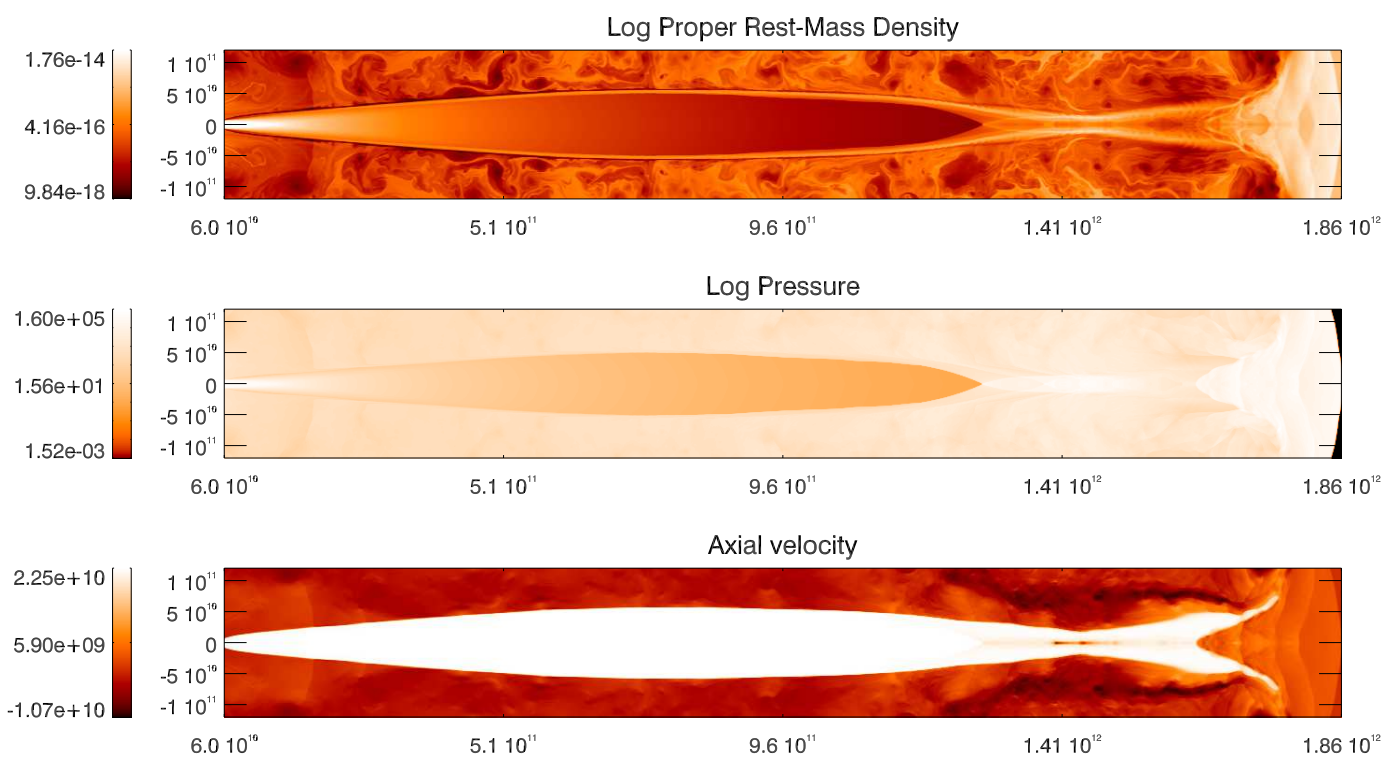

Fig. 2. Maps of rest mass density $\left(\mathrm{g} \mathrm{cm}^{-3}\right)$, pressure $(\mathrm{dyn})$ and axial velocity $(\mathrm{cm} / \mathrm{s})$ at the end of the simulation $\left(t_{\mathrm{f}}=212 \mathrm{~s}\right)$, for a powerful jet. We have included the map of axial velocity in order to illustrate the jump in velocity at the recollimation shock.

(2008).

13. Khangulyan, D., Aharonian, F. A., Bosch-Ramon, V. MNRAS in press [astro-ph/0707.1689] (2007).

14. Aharonian, F.A. et al., Science 309, 746 (2005).

15. Albert, J. et al., Science 312, 1771 (2006).
16. Albert, J. et al., ApJ Letters 665, 51 (2007).

17. Romero G.E., Torres, D. F., Kaufman Bernadó, M. M., \& Mirabel, I. F. A\&A, 410, L1 (2003)

18. Aharonian, F. A., Anchordoqui, L. A., Khangulyan, D., Montaruli, T. J. Phys. Conf. Ser., 39, 408 (2006) 